\title{
The Global Stability Analysis of a Mathematical Cellular Model of Hepatitis C Virus Infection with Non-Cytolytic Process
}

\author{
Alexis Nangue ${ }^{1}$, Cyprien Fokoue ${ }^{2}$, Raoue Poumeni' \\ ${ }^{1}$ Department of Mathematics, Higher Teachers' Training College, University of Maroua, Maroua, Cameroon \\ ${ }^{2}$ Department of Mathematics and Computer Science, Faculty of Science, University of Maroua, Maroua, Cameroon \\ Email: alexnanga02@yahoo.fr, cyprienjojofokoue@gmail.com, poumeniraoue@gmail.com
}

How to cite this paper: Nangue, A., Fokoue, C. and Poumeni, R. (2019) The Global Stability Analysis of a Mathematical Cellular Model of Hepatitis C Virus Infection with Non-Cytolytic Process. Journal of Applied Mathematics and Physics, 7, 1531-1546.

https://doi.org/10.4236/jamp.2019.77104

Received: May 28, 2019

Accepted: July 21, 2019

Published: July 24, 2019

Copyright $\odot 2019$ by author(s) and Scientific Research Publishing Inc. This work is licensed under the Creative Commons Attribution International License (CC BY 4.0).

http://creativecommons.org/licenses/by/4.0/

(c) (i) Open Access

\begin{abstract}
The aim of this work is to analyse the global dynamics of an extended mathematical model of Hepatitis $\mathrm{C}$ virus (HCV) infection in vivo with cellular proliferation, spontaneous cure and hepatocyte homeostasis. We firstly prove the existence of local and global solutions of the model and establish some properties of this solution as positivity and asymptotic behaviour. Secondly we show, by the construction of appropriate Lyapunov functions, that the uninfected equilibrium and the unique infected equilibrium of the mathematical model of HCV are globally asymptotically stable respectively when the threshold number $\mathcal{R}_{0}<1-\frac{q}{d_{I}+q}$ and when $\mathcal{R}_{0}>1$.
\end{abstract}

\section{Keywords}

HCV Model, Global Solutions, Non-Cytolytic Process, Invariant Set, Lyapunov Functions, Basic Reproduction Number, Equilibrium Points

\section{Introduction}

According to [1] [2], approximately 200 million people worldwide are persistently infected with the hepatitis $\mathrm{C}$ virus (HCV) and are at risk of developing chronic liver disease, cirrhosis, and hepatocellular carcinoma. HCV infection therefore represents a significant global public health problem. HCV establishes chronic hepatitis in $60 \%-80 \%$ of infected adults [3]. A vaccine against infection with HCV does not exist yet, and standard treatment with interferon- $\alpha$ and ribavirin has produced sustained virological response rates of approximately $50 \%$, with no effective alternative treatment for nonresponders to this treatment pro- 
tocol. A model of human immunodeficiency virus infection was adapted by Neumann et al. [4] to study the kinetics of chronic HCV infection during treatment and some mathematical analysis was done by [5]. Since then viral kinetics modeling has played an important role in the analysis of HCV RNA decay during antiviral therapy (see Perelson [6], Perelson et al. [7]). The original Neumann et al. model for $\mathrm{HCV}$ infection included three differential equations representing the populations of target cells, productively infected cells, and virus. In this paper we are going to study global dynamics of an HCV infection mathematical model with full logistic terms, antivirus treatments and homeostasis phenomenon. A similar work has been done by A. Nangue [8] concerning a mathematical intracellular HCV infection model with therapy.

\subsection{The Compartmental Model}

There are too many mathematical models of HCV dynamics amongst those, the original model or model of Newmann [4] and its extended models as those in [5] [9] for example. Each model can be represented by a compartmental scheme. A compartmental scheme is a scheme for estimating the variation in the number of individuals in each compartment over time. Figure 1 is the schematic representation of the extended model, which we will study, of HCV with cellular proliferation and spontaneous healing designed by T. C. Reluga et al. [10]. This model expands the viral dynamics of the original model of infection and the disappearance of HCV by incorporating the proliferation and death density dependence. In addition to cell proliferation, the number of uninfected hepatocytes may increase through immigration or differentiation of hepatocyte precursors that develop into hepatocytes at a constitutive rate of $s$ or by spontaneous infected

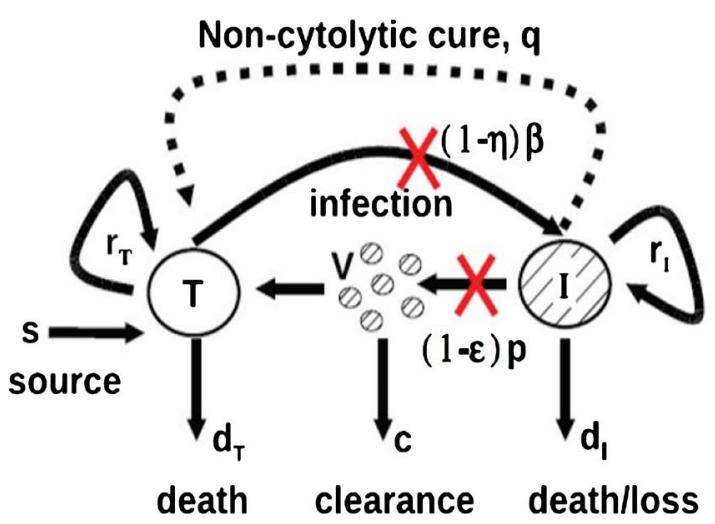

Figure 1. Schematic representation of HCV infection models. $T$ and $I$ represent target and infected cells, respectively, and $V$ represents free virus. The parameters shown in the figure are defined in the text. The original model of Neumann et al. [4] assumed that there is no proliferation of target and infected cells (i.e. $\left.r_{T}=r_{I}=0\right)$ and no spontaneous cure (i.e. $q=0$ ). The extended model of Dahari, Ribeiro, and Perelson [11], which was used for predicting complex HCV kinetics under therapy, includes target and infected cell proliferation without cure $\left(r_{T}>0, r_{I}>0\right.$ and $\left.q=0\right)$. A model including both proliferation and the spontaneous cure of infected cells (dashed line; $q>0$ ) was used to explain the kinetics of HCV in primary infection in chimpanzees [12]. 
hepatocyte healing by a non-cytolytic process at the rate $q$.

The model proposed by Dahari and coworkers [11] [13] expands on the standard HCV viral-dynamic model [4] of infection and clearance by incorporating density-dependent proliferation and death. Uninfected hepatocytes or noninfected hepatocytes, $T$, are infected at a rate $\beta$ per free virus per hepatocyte. Infected cells, $I$, produce free virus at rate $p$ per cell but also die with rate $d_{I}$. Free virus, $V$, is cleared at rate $c$ by immune and other degradation processes. Besides infection processes, hepatocyte numbers are influenced by homeostatic processes. Uninfected hepatocytes die at rate $d_{T}$. Both infected and uninfected hepatocytes proliferate logistically with maximum rates $r_{I}$ and $r_{T}$, respectively, as long as the total number of hepatocytes is less than $T_{\max }$. Besides proliferation, uninfected hepatocytes may increase in number through immigration or differentiation of hepatocyte precursors that develop into hepatocytes at constitutive rate $s$, or by spontaneous cure of infected hepatocytes through a noncytolytic process at rate $q$. Treatment with antiviral drugs reduces the infection rate by a fraction $\eta$ and the viral production rate by a fraction $\varepsilon$. It should be noted that $\eta$ and $\varepsilon$ are parameters which values are non-negative and less than one. A further comprehensive survey on the description of the model is given in [10] [14] [15]. Given the meanings of $\eta$ and $\beta$, the term $(1-\eta) \beta V T$ represents the mass action principle; $\beta V T$ is the rate of infection of healthy hepatocytes T by interaction with virus $V$.

\subsection{The Mathematical Model}

According to Reluga et al. [10] and more precisely according to the schematic representation of $\mathrm{HCV}$ infection model in Figure 1 we have the following dynamics:

- the variation of the healthy hepatocytes or uninfected hepatocytes, $T$, is expressed by the following equation:

$$
\begin{aligned}
\frac{\mathrm{d} T}{\mathrm{~d} t}= & s+r_{T} T\left(1-\frac{T+I}{T_{\max }}\right)-d_{T} T \\
& -(1-\eta) \beta V T+q I .
\end{aligned}
$$

- the variation of infected hepatocytes, $I$, is expressed by the following equation:

$$
\begin{aligned}
\frac{\mathrm{d} I}{\mathrm{~d} t}= & r_{I} I\left(1-\frac{T+I}{T_{\max }}\right)-d_{I} I \\
& -(1-\eta) \beta V T-q I .
\end{aligned}
$$

- the variation of free virions or virus, $V$, is expressed by the following equation:

$$
\frac{\mathrm{d} V}{\mathrm{~d} t}=(1-\varepsilon) p I-c V .
$$

Thus, the phenomenon described above is governed by the following mathematical model (4), which is a system of three differential autonomous equations: 


$$
\left\{\begin{array}{l}
\frac{\mathrm{d} T}{\mathrm{~d} t}=s+r_{T} T\left(1-\frac{T+I}{T_{\max }}\right)-d_{T} T-(1-\eta) \beta V T+q I ; \\
\frac{\mathrm{d} I}{\mathrm{~d} t}=r_{I} I\left(1-\frac{T+I}{T_{\max }}\right)-d_{I} I-(1-\eta) \beta V T-q I ; \\
\frac{\mathrm{d} V}{\mathrm{~d} t}=(1-\varepsilon) p I-c V
\end{array}\right.
$$

To analyse the system (4) we need the following initial conditions:

$$
T_{0}=T\left(t_{0}\right), I_{0}=I\left(t_{0}\right) \text { and } V_{0}=V\left(t_{0}\right) \text { where } t_{0} \in[0,+\infty[\text {. }
$$

For biological significance of the parameters, three assumptions are employed. (a) Due to the burden of supporting virus replication, infected cells may proliferate more slowly than uninfected cells, i.e. $r_{I} \leq r_{T}$. (b) To have a physiologically realistic model, in an uninfected liver when $T_{\max }$ is reached, liver size should no longer increase, i.e. $s \leq d_{T} T_{\max }$. (c) Infected cells have a higher turnover rate than uninfected cells, i.e. $d_{I} \geq d_{T}$. The interpretations and biologically plausible values of other parameters and a further comprehensive survey on the description of (4) is given in [10]. Besides HCV infection, the similar model of (4) is also used to describe the dynamics of HBV or HIV infection, in which the full logistic terms mean the proliferation of uninfected/infected hepatocytes [12] [16] [17], or the mitotic transmission of uninfected/infected $\mathrm{CD}^{4+} \mathrm{T}$ cells.

Our goal is therefore to analyze the stability of an extended model of HCV infection in a patient with cell proliferation and spontaneous healing given by (4) to reveal significant information on pathogenesis and dynamics of this virus. The paper is organized as follows: In Section 1, first focuses on some properties of the solutions of the model, then we calculate the basic reproduction ratio $\mathcal{R}_{0}$, which is an indispensable element in the study and analysis of the models. We theoretically analyze the local stability where we widely use the works of A. Nangue et al. [18] in Section 2. In Section 3 we theoretically analyze with some assumptions the global stability of the model by constructing appropriate Lyapounov's functions.

\section{Properties of Solutions to the Initial Value Problem (4),} (5)

\subsection{Positivity, Global Solutions and Asymptotic Behaviour}

Theorem 1. Let $T_{0}, I_{0}, V_{0} \in \mathbb{R}$. There exists $t_{1}>0$ and functions $T, I, V:\left[t_{0} ; t_{1}[\rightarrow \mathbb{R}\right.$ continuously differentiable such that $(T, I, V)$ is a solution of system (1) satisfying (4).

Theorem 2. Let $(T, I, V)$ be a solution of the system (1) over an interval $\left[t_{0}, t_{1}\left[\right.\right.$ such that $T\left(t_{0}\right)=T_{0}, I\left(t_{0}\right)=I_{0}$ et $V\left(t_{0}\right)=V_{0}$.

If $T_{0}, I_{0}, V_{0}$ are positive, then $T(t), I(t)$ and $V(t)$ are also positive for all $t \in\left[t_{0}, t_{1}[\right.$.

Proof. We are going to prove by contradiction. so suppose there is $t \in\left[t_{0}, t_{1}[\right.$ such that $T(t)=0$ or $I(t)=0$ or $V(t)=0$. 
Let $x=\left(x_{1}, x_{2}, x_{3}\right)=(T, I, V)$

Let also $t_{*}$ be the smallest of all $t$ in the interval $\left[t_{0}, t_{1}\left[\right.\right.$ such that $x_{i}(t)>0$, $\forall t \in\left[t_{0}, t_{*}\left[, \forall t \in\left[t_{0}, t_{*}\left[\right.\right.\right.\right.$ and $x_{i}\left(t_{*}\right)=0$ for a certain $i$.

Then each of the equations of the system (4) can be written $\dot{x}_{i}=-h_{i}(x)+g_{i}(x)$ where $g_{i}$ is a non negative function and $h_{i}$ any function. As a consequence $\frac{\mathrm{d} x_{i}(t)}{\mathrm{d} t} \geq-x_{i} f(x)$ and $x_{i}(t)>0, \forall t \in\left[t_{0}, t_{*}[\right.$. A contradiction.

Theorem 3. [18] The solutions of the Cauchy problem (4), (5), with positive initial data, exist globally in time in the future that is on $\left[t_{0},+\infty[\right.$.

Theorem 4. For any positive solution $(T, I, V)$ of system (4), (5) we have:

$$
T(t) \leq \tilde{T}_{0}, I(t) \leq \tilde{T}_{0} \text { and } V(t) \leq \lambda_{0}
$$

where

$$
\tilde{T}_{0}=\frac{T_{\max }}{2 r_{I}}\left(\sqrt{\left(r_{T}-d_{T}\right)^{2}+\frac{4 s r_{I}}{T_{\max }}}+r_{T}-d_{T}\right), \lambda_{0}=\lambda_{0}=\max \left\{V_{0}, \frac{1-\varepsilon}{c} p \tilde{T}_{0}\right\} .
$$

Proof. Summing Equations (6) and (7), we get:

$$
\begin{aligned}
& \frac{\mathrm{d}}{\mathrm{d} t}(T+I)=s+\left(1-\frac{T+I}{T_{\max }}\right)\left(r_{T} T-r_{I} I\right)-d_{T} T-d_{I} I \\
& =s+\left(r_{T}-d_{T}\right) T+\left(r_{I}-d_{I}\right) I-\frac{T+I}{T_{\max }}\left(r_{T} T+r_{I} I\right) \\
& \leq s+\left(r_{T}-d_{T}\right)(T+I)-\frac{T+I}{T_{\max }}\left(r_{T} T+r_{I} I\right) \text { since } r_{T}-d_{T} \geq r_{I}-d_{I},
\end{aligned}
$$

thus $\frac{\mathrm{d}}{\mathrm{d} t}(T+I) \leq s+\left(r_{T}-d_{T}\right)(T+I)-\frac{r_{I}}{T_{\max }}(T+I)^{2}$ since $r_{I} \leq r_{T}$.

Let $N_{1}=T+I, a=s>0, \quad b=\left(r_{T}-d_{T}\right)>0, \quad d=-\frac{r_{I}}{T_{\max }}<0$ and let us solve the following equation

$$
\frac{\mathrm{d} N_{1}}{\mathrm{~d} t}=a+b N_{1}+d N_{1}^{2}
$$

Coupled to Equation (6) the initial condition:

$$
N_{1}\left(t_{0}\right)=N_{1}^{0} .
$$

The solving of the problem (6), (7) gives for all $t \in\left[t_{0},+\infty[\right.$,

$$
\begin{aligned}
N_{1}(t)= & -\frac{1}{2 d}\left[\operatorname { t a n h } \left(\frac{1}{2} \sqrt{-4 a d+b^{2}}-\frac{1}{2} t_{0} \sqrt{-4 a d+b^{2}}\right.\right. \\
& \left.\left.-\arctan \left(\frac{2 N_{1}^{0}+b}{\sqrt{-4 a d+b^{2}}}\right)\right) \sqrt{-4 a d+b^{2}}\right]-\frac{b}{2 d} .
\end{aligned}
$$

As for all $x \in \mathbb{R},-1 \leq \tanh x \leq 1$, it follows that:

$$
N_{1}(t) \leq \frac{T_{\max }}{2 r_{I}}\left(\sqrt{\left(r_{T}-d_{T}\right)^{2}+\frac{4 s r_{I}}{T_{\max }}}+r_{T}-d_{T}\right) .
$$

Let 


$$
\tilde{T}_{0}=\frac{T_{\max }}{2 r_{I}}\left(\sqrt{\left(r_{T}-d_{T}\right)^{2}+\frac{4 s r_{I}}{T_{\max }}}+r_{T}-d_{T}\right) .
$$

Therefore

$$
T+I \leq \tilde{T}_{0}
$$

Since $\mathrm{T}$ and $\mathrm{I}$ are positive $I \leq T+I$ and $T \leq T+I$, so it follows that $T(t) \leq \tilde{T}_{0}$ and $I(t) \leq \tilde{T}_{0}$.

Equation (3), according to Gromwall inequality, leads to:

$$
V(t) \leq \lambda_{0} \text {. }
$$

where

$$
\lambda_{0}=\max \left\{V_{0}, \frac{1-\varepsilon}{c} p \tilde{T}_{0}\right\} .
$$

This completes the proof of theorem 4.

\subsection{Basic Reproduction Ratio $\mathcal{R}_{0}$, Invariant Set of the Model and Equilibria}

Proposition 5. The uninfected equilibrium point $E^{0}$ of the system (4) is given by

$$
E^{0}=\left(T^{0}, 0,0\right)
$$

where:

$$
T^{0}=\frac{T_{\max }}{2 r_{T}}\left(r_{T}-d_{T}+\sqrt{\left(r_{T}-d_{T}\right)^{2}+\frac{4 r_{T} s}{T_{\max }}}\right) .
$$

We use the method proposed in [19] [20] to compute the basic reproduction number $\mathcal{R}_{0}$.

Proposition 6. The expression of the basic reproduction number $\mathcal{R}_{0}$ associated to the system (4) is given by.

$$
\mathcal{R}_{0}=\frac{r_{I}}{d_{I}+q}\left(1-\frac{T^{0}}{T_{\max }}\right)+\frac{(1-\theta) \beta T^{0} p}{c\left(d_{I}+q\right)} .
$$

where

$$
1-\theta=(1-\varepsilon)(1-\eta)
$$

Remark 1. $\theta \in] 0,1[$ denotes the overall effectiveness rate of the drug.

Remark 2. Henceforth, we will let $\delta=d_{I}+q$ and $1-\theta=(1-\varepsilon)(1-\eta)$.

Theorem 7. Let $\left(t_{0}, S_{0}=\left(T_{0}, I_{0}, V_{0}\right)\right) \in R \times R_{+}^{3}$ and $\left(\left[t_{0}, T[, S=(T, I, V))\right.\right.$ be a maximal solution of the Cauchy problem (1), (4) $(T \in] t_{0},+\infty[)$. If $T\left(t_{0}\right)+I\left(t_{0}\right) \leq \tilde{T}_{0}$ and $V\left(t_{0}\right) \leq \lambda_{0}$ then the set:

$$
\Omega=\left\{(T, I, V) \in \mathbb{R} ; 0<T+I \leq \tilde{T}_{0} ; 0<V \leq \lambda_{0}\right\},
$$

where:

$$
\tilde{T}_{0}=\frac{T_{\max }}{2 r_{I}}\left(\sqrt{\left(r_{T}-d_{T}\right)^{2}+\frac{4 s r_{I}}{T_{\max }}}+r_{T}-d_{T}\right) \text { and } \lambda_{0} \max \left\{V_{0}, \frac{1-\varepsilon}{c} p \tilde{T}_{0}\right\},
$$


is a positively invariant set by system (4).

When it exists, the infected equilibrium point is given by: $E^{*}=\left(T^{*}, I^{*}, V^{*}\right)$ where $T^{*}, I^{*}$ and $V^{*}$ are positive constants that we are going to determine.

Lemma 1. [18] $T^{*}$ exists if and only if

$$
s+q \frac{T_{\max }}{r_{I}}\left(r_{I}-\delta\right)>0 .
$$

Lemma 2. [18] When it exists, $T^{*}$ is defined by:

$$
T^{*}=\frac{1}{2}\left(-\frac{D}{H}+\sqrt{\left(\frac{D}{H}\right)^{2}+F+\frac{4 s T_{\max }}{r_{T}} H}\right)
$$

where:

$$
\begin{gathered}
D=A T_{\max }\left(\frac{1}{r_{T}}\left(1+\frac{d_{T}+q}{A}\right)-\frac{\delta}{r_{I}}\left(\frac{1}{r_{T}}+\frac{1}{A}\right)-\frac{q}{r_{T} r_{I}}\right) ; \\
F=\frac{4 A q T_{\max }^{2}}{H^{2} r_{T}^{2} r_{I}^{2}}\left(A\left(\delta-r_{I}\right)-d_{I}\left(r_{I}-r_{T}\right)-r_{I}\left(q-r_{I}-r_{T}\right)+r_{T} q\right) ;
\end{gathered}
$$

and

$$
H=\frac{A^{2}}{r_{I} r_{T}}+\frac{A}{r_{I}}-\frac{A}{r_{T}} ; A=\frac{(1-\theta) \beta p T_{\max }}{C}
$$

The combination of the lemma 1 and the lemma 2 leads to the following theorem:

Theorem 8. The model(4) admits a unique infected equilibrium $E^{*}=\left(T^{*}, I^{*}, V^{*}\right)$ if and only if $\mathcal{R}_{0}>1$, where

$$
\begin{gathered}
T^{*}=\frac{1}{2}\left(-\frac{D}{H}+\sqrt{\left(\frac{D}{H}\right)^{2}+F+\frac{4 s T_{\max }}{r_{T}} H}\right), \\
I^{*}=T^{*}\left(\frac{A}{r_{I}}-1\right)+T_{\max }\left(1-\frac{\delta}{r_{I}}\right), \\
V^{*}=\frac{(1-\varepsilon) p I^{*}}{c} ;
\end{gathered}
$$

When $\mathcal{R}_{0} \leq 1$ the unique equilibrium is the uninfected equilibrium point or the infection-free steady state $E^{0}=\left(T^{0}, 0,0\right)$.

\section{Local Stability Analyses}

\subsection{Case of the Uninfected Equilibrium Point or Infection-Free Steady State}

Theorem 9. The infection-free steady state $E^{0}=\left(T^{0}, 0,0\right)$ of model (4) is locally asymptotically stable if $\mathcal{R}_{0} \leq 1$ and unstable if $\mathcal{R}_{0}>1$.

Proof. See the appendice of [18].

\subsection{Case of Infected Equilibrium Point}

We start this section by this lemma where the proof can be found in [18]. 
Lemma 3 The characteristic equation of the Jacobian matrix $J\left(E^{*}\right)$ of the system (4) at $E^{*}$ is given by the following cubic equation:

$$
\lambda^{3}+A_{1} \lambda^{2}+A_{2} \lambda+A_{3}=0
$$

where:

$$
\begin{aligned}
A_{1}= & c+\frac{s}{T^{*}}+\frac{r_{T} T^{*}+r_{I} I^{*}+A T^{*}}{T_{\max }}+q \frac{I^{*}}{T^{*}}, \\
A_{2}= & \frac{c S}{T^{*}}+\frac{c r_{T} T^{*}+s A+c r_{I} I^{*}}{T_{\max }}+q \frac{I^{*}}{T^{*}}\left(r_{I}-\delta\right)+\frac{s r_{I} I^{*}}{T^{*} T_{\max }} \\
& +\frac{r_{T} A T^{*}\left(T^{*}+I^{*}\right)}{T_{\max }^{2}}+\frac{c q I^{*}}{T^{*}}+q \frac{I^{*}}{T_{\max }}, \\
A_{3}= & \frac{c S r_{I} I^{*}}{T^{*} T_{\max }}+\frac{c A^{2} I^{*} T^{*}}{T_{\max }^{2}}-\frac{c A r_{I} I^{*} T^{*}}{T_{\max }^{2}}+\frac{c A r_{T} I^{*} T^{*}}{T_{\max }^{2}}+q \frac{c I^{*}}{T^{*}}\left(r_{I}-\delta\right) .
\end{aligned}
$$

Proof. See [18].

Now let:

$$
\Delta_{2}=\left|\begin{array}{ll}
A_{1} & 1 \\
A_{3} & A_{2}
\end{array}\right|
$$

According to lemma 3 combined with the Routh-Hurwitz criterion [21], we have the following results where the proofs can be found in [18].

Theorem 10. For model (4), when $\mathcal{R}_{0}>1$ is valid, the unique endemic equilibrium $E^{*}$ is locally asymptotically stable if $\Delta_{2}>0$ and unstable if $\Delta_{2}<0$.

Especially, we have:

Corollary 1. The infected steady state during the therapy $E^{*}$ of the model (4) is locally asymptotically stable if $\mathcal{R}_{0}>1$ and unstable if $\mathcal{R}_{0}>1$.

\section{Global Stability Analyses}

The global stability analysis of a dynamical system is usually a very complex problem. One of the most efficient methods to solve this problem is Lyapunov's theory. To build the functions of Lyapunov we will follow the method proposed by A. Korobeinikov [22] [23] [24]. In the proofs of the results that follow, to simplify the writings, we can use differently $\frac{\mathrm{d}}{\mathrm{d} t}$ or $\cdot$ for the derivation with respect to time.

\subsection{Case of Infection-Free Steady State}

Theorem 11. The infection-free steady state $E^{0}=\left(T^{0}, 0,0\right)$ of the model (4) is globally asymptotically stable if the basic reproduction number $R_{0}<1-\frac{q}{\delta}$ and unstable if $R_{0}>1-\frac{q}{\delta}$.

Proof. Consider the Lyapunov function: 


$$
L(T, I, V)=T-T^{0}-T^{0} \ln \frac{T}{T^{0}}+I+\frac{(1-\eta) \beta T^{0}}{c} V .
$$

$L$ is defined, continuous and positive definite for all $T>0, I>0, V>0$. Also, the global minimum $L=0$ occurs at the infection free equilibrium $E^{0}$. Further, function $L$, along the solutions of system (4), satisfies:

$$
\begin{aligned}
\frac{\mathrm{d} L}{\mathrm{~d} t}= & \frac{\partial L}{\partial T} \frac{\mathrm{d} T}{\mathrm{~d} t}+\frac{\partial L}{\partial I} \frac{\mathrm{d} I}{\mathrm{~d} t}+\frac{\partial L}{\partial V} \frac{\mathrm{d} V}{\mathrm{~d} t} \\
= & \left(1-\frac{T^{0}}{T}\right) \dot{T}+\dot{I}+\frac{(1-\eta) \beta T^{0}}{c} \dot{V} \\
= & \left(T-T^{0}\right) \frac{\dot{T}}{T}+\dot{I}+\frac{(1-\eta) \beta T^{0}}{c} \dot{V} \\
= & \left(T-T^{0}\right)\left(\frac{s}{T}+r_{T}-\frac{r_{T}(T+I)}{T_{\max }}-\mathrm{d} T-(1-\eta) \beta V+q \frac{I}{T}\right)+(1-\eta) \beta V T \\
& +r_{I} I\left(1-\frac{T+I}{T_{\max }}\right)-\delta I+\frac{1-\theta}{c} \beta p T^{0} T-(1-\eta) \beta T^{0} V \\
= & \left(T-T^{0}\right)\left(\frac{s}{T}+r_{T}-d T-\frac{r_{T}(T+I)}{T_{\max }}+q \frac{I}{T}\right)-T(1-\eta) \beta V+T^{0}(1-\eta) \beta V \\
+ & (1-\eta) \beta V T+r_{I} I\left(1-\frac{T+I}{T_{\max }}\right)-\delta I+\frac{1-\theta}{c} \beta p T^{0} I-(1-\eta) \beta T^{0} V,
\end{aligned}
$$

i.e.

$$
\begin{aligned}
\frac{\mathrm{d} L}{\mathrm{~d} t}= & \left(T-T^{0}\right)\left(\frac{s}{T}+r_{T}-d T-\frac{r_{T}(T+I)}{T_{\max }}\right)+q I-q I \frac{T^{0}}{T} \\
& +r_{I} I\left(1-\frac{T+I}{T_{\max }}\right)+\left(\frac{1-\theta}{c} \beta p T^{0}-\delta\right) I ;
\end{aligned}
$$

yet

$$
r_{T}-d_{T}=\frac{r T^{0}}{T_{\max }}-\frac{s}{T^{0}}
$$

hence, Further collecting terms, we have:

$$
\begin{aligned}
\frac{\mathrm{d} L}{\mathrm{~d} t}= & \left(T-T^{0}\right)\left(\frac{s}{T}+\frac{r T^{0}}{T_{\max }}-\frac{s}{T^{0}}-\frac{r_{T}(T+I)}{T_{\max }}\right) \\
& +r_{I} I\left(1-\frac{T+I}{T_{\max }}\right)+\left(\frac{1-\theta}{c} \beta p T^{0}-\delta\right) I \\
= & \left(T-T^{0}\right)\left(-\frac{s}{T T^{0}}\left(T-T^{0}\right)-\frac{r_{T}}{T_{\max }}\left(T-T^{0}\right)-\frac{r_{T} I}{T_{\max }}\right) \\
& +r_{I} I\left(1-\frac{T+I}{T_{\max }}\right)+\left(\frac{1-\theta}{c} \beta p T^{0}-\delta\right) I+q I-q I \frac{T^{0}}{T} \\
= & -\frac{s}{T T^{0}}\left(T-T^{0}\right)^{2}-\frac{r_{T}}{T_{\max }}\left(\left(T-T^{0}\right)^{2}+\left(T-T^{0}\right) I\right)+r_{I} I-\frac{r_{I} I T}{T_{\max }} \\
& -\frac{r_{I} I^{2}}{T_{\max }}+\left(\frac{1-\theta}{c} \beta p T^{0}-\delta\right) I+q I-q I \frac{T^{0}}{T}
\end{aligned}
$$




$$
\begin{aligned}
= & -\frac{s}{T T^{0}}\left(T-T^{0}\right)^{2}-\frac{r_{T}}{T_{\max }}\left(\left(T-T^{0}\right)^{2}+\left(T-T^{0}\right) I+\frac{r_{I} I T}{r_{T}}+\frac{r_{I} I^{2}}{r_{T}}\right) \\
& +r_{I} I+\left(\frac{1-\theta}{c} \beta p T_{0}-\delta\right) I+q I-q I \frac{T^{0}}{T} \\
= & -\frac{s}{T T_{0}}\left(T-T^{0}\right)^{2}-\frac{r_{T}}{T_{\max }}\left[\left(T-T^{0}\right)^{2}+\left(T-T^{0}\right) I+\frac{r_{I} I T}{r_{T}}+\frac{r_{I} I^{2}}{r_{T}}\right. \\
& \left.+\frac{r_{I}}{r_{T}} I T^{0}-\frac{r_{I}}{r_{T}} I T^{0}\right]+r_{I} I+\left(\frac{1-\theta}{c} \beta p T^{0}-\delta\right) I+q I-q I \frac{T^{0}}{T} \\
= & -\frac{s}{T T^{0}}\left(T-T^{0}\right)^{2}-\frac{r_{T}}{T_{\max }}\left(\left(T-T^{0}\right)^{2}+\left(T-T^{0}\right) I+\frac{r_{I} I T}{r_{T}}\left(T-T^{0}\right)\right. \\
& \left.+\frac{r_{I} I^{2}}{r_{T}}+\frac{r_{I}}{r_{T}} I T^{0}\right)+r_{I} I+\left(\frac{1-\theta}{c} \beta p T^{0}-\delta\right) I+q I-q I \frac{T^{0}}{T} \\
= & -\frac{s}{T T^{0}}\left(T-T^{0}\right)^{2}-\frac{r_{T}}{T_{\max }}\left(T+I-T^{0}\right)\left(T+\frac{r_{I}}{r_{T}} I-T^{0}\right) \\
& -\frac{r_{I}}{T_{\max }} I T^{0}+r_{I} I+\left(\frac{1-\theta}{c} \beta p T^{0}-\delta\right) I+q I-q I \frac{T^{0}}{T} \\
= & -\frac{s}{T T^{0}}\left(T-T^{0}\right)^{2}-\frac{r_{T}}{T_{\max }}\left(T+I-T^{0}\right)\left(T+\frac{r_{I}}{r_{T}} I-T^{0}\right) \\
& +\delta I\left(\frac{r_{I}}{\delta}-\frac{r_{I} T^{0}}{\delta T_{\max }} \frac{1-\theta}{c \delta} \beta p T^{0}-1\right)+q I-q I \frac{T^{0}}{T} .
\end{aligned}
$$

Furthermore,

$$
\mathcal{R}_{0}=\frac{1-\theta}{c \delta} \beta p T^{0}+\frac{r_{I}}{\delta}\left(1-\frac{T^{0}}{T_{\max }}\right),
$$

hence

$$
\begin{aligned}
\frac{\mathrm{d} L}{\mathrm{~d} t}= & -\frac{s}{T T^{0}}\left(T-T^{0}\right)^{2}-\frac{r_{T}}{T_{\max }}\left(T+I-T^{0}\right)\left(T+\frac{r_{I}}{r_{T}} I-T^{0}\right) \\
& -q I \frac{T^{0}}{T}+\delta I\left(\mathcal{R}_{0}-1\right)+q I \\
= & -\frac{s}{T T^{0}}\left(T-T^{0}\right)^{2}-\frac{r_{T}}{T_{\max }}\left(T+I-T^{0}\right)\left(T+\frac{r_{I}}{r_{T}} I-T^{0}\right) \\
& -q I \frac{T^{0}}{T}+\delta I\left(\mathcal{R}_{0}-1+\frac{q}{\delta}\right) .
\end{aligned}
$$

Since $r_{I} \leq r_{T}$ and $\mathcal{R}_{0}<1-\frac{q}{\delta}$, we have $\frac{\mathrm{d} L}{\mathrm{~d} t} \leq 0$ and $\frac{\mathrm{d} L}{\mathrm{~d} t}=0$ if and only if $T=T^{0}$ and $I=0$ simultaneously.

Therefore, the largest compact invariant subset of the set

$$
M=\left\{(T, I, V) \in \Omega: \frac{\mathrm{d} L}{\mathrm{~d} t}=0\right\}
$$


is the singleton $\left\{E^{0}\right\}$. By the Lasalle invariance principle [25], the infection-free equilibrium is globally asymptotically stable if $\mathcal{R}_{0}<1-\frac{q}{\delta}$. We have seen previously that if $\mathcal{R}_{0}>1$, at least one of the eigenvalues of the Jacobian matrix evaluated at $E^{0}$ has a positive real part. Therefore, the infection-free equilibrium $E^{0}$ is unstable when $\mathcal{R}_{0}>1$. This completes the proof of the theorem.

Remark 3. The Lyapunov function defined in the proof of theorem 11 has been obtained following the general form giving by Korobonikov [22] [23] [24] for the dynamic virus fondamental model.

\subsection{Case of Infected Equilibrium Point}

We recall:

Remark 4. The infected equilibrium point $E^{*}=\left(T^{*}, I^{*}, V^{*}\right)$ satisfies:

$$
\begin{gathered}
r_{T}-d_{T}=-\frac{s}{T^{*}}+(1-\eta) \beta V^{*}+\frac{r_{T}}{T_{\max }}\left(T^{*}+I^{*}\right)-q \frac{I^{*}}{T^{*}}, \\
r_{I}-\delta=-\frac{(1-\eta) \beta V^{*} T^{*}}{I^{*}}+\frac{r_{I}}{T_{\max }}\left(T^{*}+I^{*}\right), \\
c=\frac{(1-\varepsilon) p I^{*}}{V^{*}} .
\end{gathered}
$$

Now we are stating and demonstrating one of the most important results of this work.

Theorem 12. Suppose that $r_{I}=r_{T}, s=d_{T} T_{\max }$ and $\delta=d_{T}$. Then the infected steady state during therapy $E^{*}$ of model (4) is globally asymptotically stable as soon as it exists.

Proof. Consider the Lyapunov function defined by:

$$
\begin{aligned}
L(T, I, V)= & T-T^{*}-T^{*} \ln \frac{T}{T^{*}}+I-I^{*}-I^{*} \ln \frac{I}{I^{*}} \\
& +\frac{(1-\eta) \beta T^{*} V^{*}}{(1-\varepsilon) p I^{*}}\left(V-V^{*}-V^{*} \ln \frac{V}{V^{*}}\right) .
\end{aligned}
$$

Let us show that $\frac{\mathrm{d} L}{\mathrm{~d} t} \leq 0$ and $\frac{\mathrm{d} L}{\mathrm{~d} t}=0$ if and only if $T=T^{*}, \quad I=I^{*}$, $V=V^{*}$ simultaneously.

The time derivative of $L$ along the trajectories of system (1) is:

$$
\begin{aligned}
\frac{\mathrm{d} L}{\mathrm{~d} t} & =\frac{\partial L}{\partial T} \frac{\mathrm{d} T}{\mathrm{~d} t}+\frac{\partial L}{\partial I} \frac{\mathrm{d} I}{\mathrm{~d} t}+\frac{\partial L}{\partial V} \frac{\mathrm{d} V}{\mathrm{~d} t} \\
& =\left(1-\frac{T^{*}}{T}\right) \dot{T}+\left(1-\frac{I^{*}}{I}\right) \dot{I}+\frac{(1-\eta) \beta T^{*} V^{*}}{(1-\varepsilon) p I^{*}}\left(1-\frac{V^{*}}{V}\right) \dot{V} \\
& =\left(T-T^{*}\right) \frac{\dot{T}}{T}+\left(I-I^{*}\right) \frac{\dot{I}}{I}+\frac{(1-\eta) \beta T^{*} V^{*}}{(1-\varepsilon) p I^{*}}\left(V-V^{*}\right) \frac{\dot{V}}{V} .
\end{aligned}
$$

Collecting terms, and canceling identical terms with opposite signs, yields: 


$$
\begin{aligned}
\frac{\mathrm{d} L}{\mathrm{~d} t}= & \left(T-T^{*}\right)\left(\frac{s}{T}+r_{T}-\frac{r_{T}(T+I)}{T_{\max }}-d_{T}-(1-\eta) \beta V+q \frac{I}{T}\right) \\
& +\frac{(1-\eta) \beta T^{*} V^{*}}{(1-\varepsilon) p I^{*}}\left(\frac{V-V^{*}}{V}\right)((1-\varepsilon) p I-c V) \\
& +\left(I-I^{*}\right)\left((1-\eta) \frac{\beta V T}{I}+r_{I}\left(1-\frac{T+I}{T_{\max }}\right)-\delta\right) .
\end{aligned}
$$

Reporting equalities (9), (10) and (11) of the remark 4 into (12), we have:

$$
\begin{aligned}
& \frac{\mathrm{d} L}{\mathrm{~d} t}=\left(T-T^{*}\right)\left[\frac{s}{T}-\frac{s}{T^{*}}+(1-\eta) \beta V^{*}+\frac{r_{T}}{T_{\max }}\left(T^{*}+I^{*}\right)-q \frac{I^{*}}{T^{*}}\right. \\
& \left.-\frac{r_{T}(T+I)}{T_{\max }}-(1-\eta) \beta V+q \frac{I}{T}\right]+\left(I-I^{*}\right)\left[(1-\eta) \frac{\beta V T}{I}\right. \\
& \left.-r_{I} \frac{T}{T_{\max }}-r_{I} \frac{I}{T_{\max }}-\frac{(1-\eta) \beta V^{*} T^{*}}{I^{*}}+\frac{r_{I}}{T_{\max }}\left(T^{*}+I^{*}\right)\right] \\
& +(1-\eta) \beta+\frac{T^{*} V^{*}}{(1-\varepsilon) p I^{*}}\left(\frac{V-V^{*}}{V}\right)\left((1-\varepsilon) p I-\frac{(1-\varepsilon) p I^{*}}{V^{*}} V\right) \\
& =-\frac{S}{T T^{*}}\left(T-T^{*}\right)^{2}-\frac{r_{T}}{T_{\max }}\left(T-T^{*}\right)^{2}-\frac{r_{T}}{T_{\max }}\left(T-T^{*}\right)\left(I-I^{*}\right) \\
& -(1-\eta) \beta\left(T-T^{*}\right)\left(V-V^{*}\right)+(1-\eta) \beta\left[\left(\frac{V T}{I}-\frac{V^{*} T^{*}}{I^{*}}\right)\left(I-I^{*}\right)\right. \\
& \left.+\frac{T^{*} V^{*}}{(1-\varepsilon) p I^{*}} \frac{V-V^{*}}{V}\left((1-\varepsilon) p I-\frac{(1-\varepsilon)}{V^{*}} p I^{*} V\right)\right]-q \frac{I^{*}}{T^{*}}\left(T-T^{*}\right) \\
& +q \frac{I}{T}\left(T-T^{*}\right)-\frac{r_{I}}{T_{\max }}\left(T-T^{*}\right)\left(I-I^{*}\right)-\frac{r_{I}}{T_{\max }}\left(I-I^{*}\right)^{2} \\
& =-\frac{S}{T T^{*}}\left(T-T^{*}\right)^{2}-\frac{r_{T}}{T_{\max }}\left(T-T^{*}\right)^{2}-\frac{r_{T}+r_{I}}{T_{\max }}\left(T-T^{*}\right)\left(I-I^{*}\right) \\
& -\frac{r_{I}}{T_{\max }}\left(I-I^{*}\right)^{2}+(1-\eta) \beta\left[\left(\frac{V T}{I}-\frac{V^{*} T^{*}}{I^{*}}\right)\left(I-I^{*}\right)\right. \\
& \left.-\left(T-T^{*}\right)\left(V-V^{*}\right)+\frac{T^{*} V^{*}}{(1-\varepsilon) p I^{*}} \frac{V-V^{*}}{V}\left((1-\varepsilon) p I-\frac{1-\varepsilon}{V^{*}} p I^{*} V\right)\right] \\
& -q \frac{1}{T T^{*}}\left(T^{2} I^{*}+\left(T^{*}\right)^{2} I-T^{*} T I-T^{*} T I^{*}\right) \\
& =-\frac{S}{T T^{*}}\left(T-T^{*}\right)^{2}-\frac{1}{T_{\max }}\left(r_{T} T+r_{I} I-r_{T} T^{*}-r_{I} I^{*}\right)\left(T+I-T^{*}-I^{*}\right) \\
& +(1-\eta) \beta T^{*} V^{*}\left(\frac{V T}{V^{*} T^{*}}-\frac{V T I^{*}}{I V^{*} T^{*}}-\frac{V^{*} T^{*} I}{I^{*} V^{*} T^{*}}+\frac{V^{*} T^{*} I^{*}}{V^{*} T^{*} I^{*}}-\frac{T V}{T^{*} V^{*}}\right. \\
& \left.+\frac{T V^{*}}{T^{*} V^{*}}+\frac{T^{*} V}{T^{*} V^{*}}-\frac{T^{*} V^{*}}{T^{*} V^{*}}+\frac{I V}{I^{*} V}-\frac{I^{*} V^{2}}{I^{*} V V^{*}}-\frac{V^{*} I}{I^{*} V}+\frac{V^{*} I^{*} V}{V^{*} I^{*} V}\right) \\
& -q \frac{1}{T T^{*}}\left(\left(T-T^{*}\right)^{2} I^{*}+\left(T^{*}\right)^{2}\left(I-I^{*}\right)+T T^{*}\left(I^{*}-I\right)\right)
\end{aligned}
$$




$$
\begin{aligned}
= & -\frac{s}{T T^{*}}\left(T-T^{*}\right)^{2}-\frac{1}{T_{\max }}\left(r_{T} T+r_{I} I-r_{T} T^{*}-r_{I} I^{*}\right)\left(T+I-T^{*}-I^{*}\right) \\
& +(1-\eta) \beta T^{*} V^{*}\left(1+\frac{T}{T^{*}}-\frac{V T I^{*}}{I V^{*} T^{*}}-\frac{V^{*} I}{I^{*} V}\right) \\
& -q \frac{1}{T T^{*}}\left(\left(T-T^{*}\right)^{2} I^{*}+T^{*}\left(I-I^{*}\right)\left(T^{*}-T\right)\right) .
\end{aligned}
$$

Note that

$$
1+\frac{T}{T^{*}}-\frac{V T I^{*}}{I V^{*} T^{*}}-\frac{V^{*} I}{I^{*} V}=\left(3-\frac{T^{*}}{T}-\frac{V T I^{*}}{I V^{*} T^{*}}-\frac{V^{*} I}{I^{*} V}\right)+\left(\frac{T}{T^{*}}+\frac{T^{*}}{T}-2\right)
$$

and

$$
\left(\frac{T}{T^{*}}+\frac{T^{*}}{T}-2\right)=\frac{\left(T-T^{*}\right)^{2}}{T T^{*}}
$$

Recall that:

$$
s=(1-\eta) \beta T^{*} V^{*}+\left(d_{T}-r_{T}+r_{T} \frac{T^{*}+I^{*}}{T_{\max }}\right) T^{*}-q I^{*} .
$$

furthermore,

$$
T^{*}+I^{*}=T_{\max }
$$

hence,

$$
s=(1-\eta) \beta T^{*} V^{*}+d_{T} T^{*}-q I^{*} .
$$

By hypothesis, $r_{T}=r_{I}$ this leads to:

$$
\begin{aligned}
& \frac{\mathrm{d} L}{\mathrm{~d} t}=\left(-\frac{d_{T}}{T}+q \frac{I^{*}}{T T^{*}-\frac{(1-\eta) \beta V^{*}}{T}}\right)\left(T-T^{*}\right)^{2}-\frac{r_{T}}{T_{\max }}\left(T+I-T^{*}-I^{*}\right)^{2} \\
& +(1-\eta) \beta V^{*} T^{*}\left(3-\frac{T^{*}}{T}-\frac{V T I^{*}}{I V^{*} T^{*}}-\frac{V^{*} I}{I^{*} V}\right)+(1-\eta) \beta V^{*} T^{*} \frac{\left(T-T^{*}\right)^{2}}{T T^{*}} \\
& -q \frac{1}{T T^{*}}\left(\left(T-T^{*}\right)^{2} I^{*}+T^{*}\left(I-I^{*}\right)\left(T^{*}-T\right)\right) \\
& =-\frac{d_{T}}{T}\left(T-T^{*}\right)^{2}-\frac{r_{T}}{T_{\max }}\left(T+I-T^{*}-I^{*}\right)^{2}-q \frac{1}{T}\left(I-I^{*}\right)\left(T^{*}-T\right) \\
& +(1-\eta) \beta V^{*} T^{*}\left(3-\frac{T^{*}}{T}-\frac{V T I^{*}}{I V^{*} T^{*}}-\frac{V^{*} I}{I^{*} V}\right) \\
& =-\frac{d_{T}}{T}\left(T-T^{*}\right)^{2}-\frac{r_{T}}{T_{\max }}\left(T+I-T^{*}-I^{*}\right)^{2}-q \frac{1}{T}\left(I-I^{*}\right)\left(T^{*}-T\right) \\
& +(1-\eta) \beta V^{*} T^{*}\left(3-\frac{\left(T^{*}\right)^{2} I I^{*} V V^{*}+\left(I^{*} V T\right)^{2}+\left(I V^{*}\right)^{2} T T^{*}}{T T^{*} I I^{*} V V^{*}}\right) \\
& =-\frac{d_{T}}{T}\left(T-T^{*}\right)^{2}-\frac{r_{T}}{T_{\max }}\left(T+I-T^{*}-I^{*}\right)^{2}-q \frac{1}{T}\left(I-I^{*}\right)\left(T^{*}-T\right) \\
& +\frac{3(1-\eta) \beta V^{*} T^{*}}{T T^{*} I I^{*} V V^{*}}\left(T T^{*} I I^{*} V V^{*}-\frac{1}{3}\left(\left(T^{*}\right)^{2} I I^{*} V V^{*}+\left(I^{*} V T\right)^{2}+\left(I V^{*}\right)^{2} T T^{*}\right)\right) \text {. }
\end{aligned}
$$


Yet

$$
\frac{1}{3}\left(\left(T^{*}\right)^{2} I I^{*} V V^{*}+\left(I^{*} V T\right)^{2}+\left(I V^{*}\right)^{2} T T^{*}\right) \geq T T^{*} I I^{*} V V^{*}
$$

since the geometric mean is less than or equal to the arithmetic mean.

$$
\text { It should be noted that } \frac{\mathrm{d} L}{\mathrm{~d} t} \leq 0 \text { and } \frac{\mathrm{d} L_{2}}{\mathrm{~d} t}=0 \text { holds if and only if }(T, I, V)
$$

take the steady states values $\left(T^{*}, I^{*}, V^{*}\right)$. Therefore, By the Lasalle invariance principle [22], the infected equilibrium point $E^{*}$ is globally asymptotically stable. This completes the proof of this theorem.

\section{Concluding Remark}

To understand the dynamics of HCV infection and its infectious processes, mathematical models are present as an important and unavoidable tool. Global stability analysis has been done, by the technique of Lyapunov, to the model of HCV infection with proliferation cell and spontaneous healing, for revealing significant information for making good decision for the fighting against hepatitis $\mathrm{C}$. This work is a starting point to many interesting other future investigations. We plan to extend our study by focusing on more realistic models such as: 1) mathematical models with delay which involve delay ordinary differential equations. 2) mathematical models taking into account space which involve Partial differential equations. 3) mathematical models taking into account random phenomena which evolve stochastic differential equations. We also plan to focus on others methods of studying global stability like the geometric method that can provide results with fewer hypotheses on mathematical model (4).

\section{Acknowledgements}

We thank the Editor and the referee for their comments. We are grateful to Professor Alan Rendall for valuable and tremendous discussions about this paper. We wish to thank him for introducing us to Mathematical Biology and to its relationship with Mathematical Analysis. We also thank the Higher Teachers' Training College of the University of Maroua were this paper were initiated.

\section{Conflicts of Interest}

The authors declare no conflicts of interest regarding the publication of this paper.

\section{References}

[1] NIH (2002) National Institutes of Health Consensus Development Conference. Management of Hepatitis C: 2002-June 10-12, 2002. Hepatology, 36, S3-S20. https://doi.org/10.1053/jhep.2002.37117

[2] WHO (2017) Global Hepatitis Repport. http://www.who.int/mediacentre/factsheets/fs164/en/

[3] Seeff, L.B. (2002) Natural History of Chronic Hepatitis C. Hepatology, 36, S35-S46. 
https://doi.org/10.1053/jhep.2002.36806

[4] Neumann, A.U., Lam, N.P., Dahari, H., Gretch, D.R., Wiley, T.E., Layden, T.J. and Perelson, A.S. (1998) Hepatitis C Viral Dynamics in Vivo and the Antiviral Efficacy of Interferon- $\alpha$ Therapy. Science, 282, 103-107.

https://doi.org/10.1126/science.282.5386.103

[5] Chong, M.S.F., Crossley, L.S.M. and Madzvamuse, A. (2015) The Stability Analyses of the Mathematical Models of Hepatitis C Virus Infection. Modern Applied Science, 9, 250-271. http://sro.sussex.ac.uk/52312/ https://doi.org/10.5539/mas.v9n3p250

[6] Perelson, A., Neumann, A., Markowitz, M., Leonard, J. and Ho, D. (1996) HIV-1 Dynamics in Vivo: Virion Clearance Rate, Infected Cell Life-Span, and Viral Generation Time. Science, 271, 1582-1586. https://doi.org/10.1126/science.271.5255.1582

[7] Perelson, A.S. (2002) Modelling Viral and Immune System Dynamics. Nature Reviews Immunology, 2, 28-36. https://doi.org/10.1038/nri700

[8] Nangue, A. (2019) Global Stabilty Analysis of a Cellular Model of Hepatitis C Virus Infection under Treatement. arXiv:1901.09984 2.

[9] Banerjee, S., Keval, R., Gakkhar, R. (2011) Modeling the Dynamics of Hepatitis C Virus with Combined Antiviral Drug Therapy: Interferon and Ribavirin. Bulletin of Mathematical Biology, arXiv:1105.3669v1.

[10] Reluga, T.C. Dahari, H. and Perelson, A.S. (2009) Analysis of Hepatitis C Virus Infection Models with Hepatocyte Homeostasis. SIAM Journal on Applied Mathematics, 69, 999-1023. http://www.siam.org/journals/ojsa.php https://doi.org/10.1137/080714579

[11] Dahari, H., Ribeiro, R.M. and Perelson, A.S. (2007) Triphasic Decline of HCV RNA during Antiviral Therapy. Hepatology, 46, 16-21. https://doi.org/10.1002/hep.21657

[12] Dahari, H., Shudo, H., Ribeiro, R.M. and Perelson, A.S. (2009) Modeling Complex Decay Profiles of Hepatitis B Virus during Antiviral Therapy. Hepatology, 49, 32-38.

[13] Daharia, H., Loa, A., Ribeiroa, R.M. and Perelson, A.S. (2007) Modeling Hepatitis C Virus Dynamics: Liver Regeneration and Critical Drug Efficacy. Journal of Theoretical Biology, 247, 371-381. http://www.sciencedirect.com https://doi.org/10.1016/j.jtbi.2007.03.006

[14] Dahari, H., Layden-Almer, J.E., Kallwitz, E., Ribeiro, R.M., Cotler, S.J., Layden, T.J. and Perelson, A.S. (2009) A Mathematical Model of Hepatitis C Virus Dynamics in Patients with High Baseline Viral Loads or Advanced Liver Disease. Gastroenterology, 136, 1402-1409. https://doi.org/10.1053/j.gastro.2008.12.060

[15] Dahari, H., Major, M., Zhang, X., Mihalik, K., Rice, C.M., Perelson, A.S., Feinstone, S.M. and Neumann, A.U. (2005) Mathematical Modeling of Primary Hepatitis C Infection: Noncytolytic Clearance and Early Blockage of Virion Production. Gastroenterology, 128, 1056-1066. https://doi.org/10.1053/j.gastro.2005.01.049

[16] Ciupe, S.M. Ribeiro, S.M. Nelson, P.W. and Perelson, A.S. (2007) Modeling the Mechanisms of Acute Hepatitis B Virus Infection. Journal of Theoretical Biology, 247, 23-35. https://doi.org/10.1016/j.jtbi.2007.02.017

[17] Li, Q., Lu, F., Deng, G. and Wang, K. (2014) Modeling the Effects of Covalently Closed Circular DNA and Dendritic Cells in Chronic HBV Infection. Journal of Theoretical Biology, 357, 1-9. https://doi.org/10.1016/j.jtbi.2014.04.037

[18] Nangue, A., Fokoue, C. and Poumeni, R. (2019) Mathematical Analysis of an Ex- 
tended Cellular Model of the Hepatitis C Virus Infection with Non-Cytolytic Process. ArXiv: $1905.02561 \mathrm{vl}$.

[19] Diekmann, O., Heesterbeek, J.P.A. and Metz, J.A.J. (1990) On the Definition and the Computation of the Basic Reproduction Ratio $R_{0}$ in Models for Infectious Diseases in Heterogeneous Populations. Journal of Mathematical Biology, 28, 365-382.

[20] Van den Driessche, P. and Watmough, J. (2002) Reproduction Numbers and Sub-Threshold Endemic Equilibria for Compartmental Models of Disease Transmission. Mathematical Biosciences, 180, 29-48. https://doi.org/10.1016/S0025-5564(02)00108-6

[21] Murray, J.M. (2001) Mathematical Biology I: An Introduction. Springer, lin, Heidelberg.

[22] Korobeinikov, A. (2004) Global Properties of Basic Virus Dynamics Models. Bulletin of Mathematical Biology, 66, 879-883. https://doi.org/10.1016/j.bulm.2004.02.001

[23] Korobeinikov, A. (2004) Lyapunov Functions and Global Properties for SEIR and SEIS Epidemic Models. Mathematical Medicine and Biology, 21, 75-83. https://doi.org/10.1093/imammb21.2.75

[24] Korobeinikov, A. and Wake, G.C. (2002) Lyapunov Functions and Global Stability for SIR, SIRS, and SIS Epidemiological Models. Applied Mathematics Letters, 15, 955-960. https://doi.org/10.1016/S0893-9659(02)00069-1

[25] Khalil, H. (2002) Nonlinear Systems. 3rd Edition, Prentice Hall, New York. 